\title{
Toxicity Assessment of Silver Nanoparticles using Zebrafish Embryos
}

\author{
Xu Qiaoshu ${ }^{1, a}$, Di Tao ${ }^{2, b}$, Zhou Xin ${ }^{2, c^{*}}$, Gu Ning ${ }^{1, d}$ \\ ${ }^{1}$ School of Biological Science and Medical Engineering, Southeast University, Nanjing 210009, \\ China. \\ ${ }^{2}$ College of Veterinary Medicine, Yangzhou University, Yangzhou 225009, China. \\ axuqiaoshu_seu@163.com, bdi_tao@126.com, 'chou_xin@126.com, dguning@seu.edu.cn
}

\section{Keywords: biological toxicity, silver nanoparticles, zebrafish embryos}

\begin{abstract}
In recent years, nanomaterials have attracted great interest from industrial community and researchers due to many reports showing that nanomaterials can be promising vehicles for in vitro diagnosis, in vivo tumor imaging and targeted therapy of many diseases in addition to being wide-spectrum antibacterial agents. Since nanomaterial can readily diffuse into aquatic environments and further enter human body, which probably pose a threat to human health thus limit the real application of nanomaterials in organism. To this end, it is very important to investigate the toxicity of nanomaterial to organisms. Herein, we evaluated the toxicity of two representative silver nanoparticles (AgNPs@PVP, AgNPs@ citrate) on the development of zebrafish (a classic model animal) embryos by incubating these silver agents and silver ions (as a positive control) with early embryos at various concentrations for 24, 48, 72 and 96 hours, respectively. The results showed that after 24 hours incubation, $\mathrm{Ag}^{+}$of $0.2 \mu \mathrm{g} / \mathrm{mL}$ led to up to $80 \%$ of mortality of embryos and AgNPs@citrate ( 15 nm diameter) can result in death of a small percentage of embryos (mortality of $20 \%)$. However, AgNPs@PVP ( 15 nm diameter) did not cause any death of embryos. As the concentration went up to $0.5 \mu \mathrm{g} / \mathrm{mL}, \mathrm{Ag}^{+}$caused $100 \%$ mortality of embryos with 48 hours incubation, and AgNPs@citrate was also highly toxic (mortality of $96 \%$ ), but the mortality rate induced by AgNPs@PVP was only $40 \%$. This data clearly indicate that the AgNPs@PVP is less toxic than AgNPs@citrate and the toxicity of AgNPs@citrate to embryos is comparable to silver ion.
\end{abstract}

\section{Introduction}

With the development of nanotechnology, silver nanoparticles, which are widely used as a broad-spectrum antibacterial reagent due to their strong no-specific antibacterial ability that even can kill as many as 650 kinds of bacteria in a few minutes, are prepared by many research labs and materials companies, [1]. Since large-scale use of silver nanoparticles-related products poses a great potential threat to the environment and human health, many researches have been carried out to explore the toxic mechanism of silver nanoparticles in recent years $[2,3,4,5]$, but there is still no clear conclusion. There are two views on the toxicity mechanism induced by silver nanoparticle. One is that silver ions are the major toxic agents and the toxicity of AgNPs is due to the release of silver ions from the nanoparticles. The other suggests that AgNPs have the Trojan horse effect which can affect organisms by entering the cell nuclear of organisms and nanoparticles can bind directly to RNA polymerase thus inhibit gene transcription by "granule effect" [6]. However, another publication reported that nanoparticles can't kill bacteria [7]. Katrine Bilberg et al. used zebrafish to investigate the toxic mechanism of silver nanoparticle [8], they proposed that silver 
ions lead to the main toxicity. Saji George also used zebrafish cells and embryos to compare the toxicity strength of different morphologies of silver nanomaterials and reported that sheet silver nanomaterials are the most toxic compared to other shape silver nanomaterials [9].

Zebrafish or zebrafish embryo toxicity test has gradually become a popular method of ecotoxicology research $[10,11,12]$. The reasons why zebrafish or zebrafish embryo is recognized as a good model organism for evaluating chemical toxicity in vivo are as follows: First, the similarity between zebrafish genes and human genes is high, which means that the results obtained from the zebrafish drug test can be potentially applied to the human body. Second, the zebrafish embryo is transparent, which makes it easy for observation. Third, the female zebrafish can lay a large number of eggs at one time, which indicating that the embryo can be easily obtained and different tests can be conducted in the same generation. In this study, we explored the toxicity of silver nanoparticles by recording the mortality rates of zebrafish embryos incubated with solution containing silver nanoparticles at various concentrations for various length of time.

\section{Materials and methods}

\section{Materials}

AgNPs@PVP and AgNPs@citrate were synthesized by a continuous flow electrochemical process as reported previously $[13,14]$. Briefly, the polished silver electrodes were fitted on the airtight container cover and then $5 \mathrm{mg} / \mathrm{mL}$ of PVP solution was injected into the container continuously by a syringe at the flowrate of $100 \mathrm{~mL} / \mathrm{h}$ at $90{ }^{\circ} \mathrm{C}$ and 15 voltages. The concentrations of the reactants were $1 \times 10^{-3} \mathrm{M}, 1 \times 10^{-2} \mathrm{M}$ and $5 \times 10^{-3} \mathrm{M}$ for $\mathrm{AgNO}_{3}$, maltose and ammonia, respectively. Sodium citrate and PVP were added separately as capping agent after the adjustment of $\mathrm{pH}$ to 11.5. Finally AgNPs@PVP and AgNPs@citrate were obtained by cleaning with deionized (DI) water followed by ultrafiltration membrane (10 $\mathrm{kDa}$ molecular weight cut-off).The zebrafish embryos were collected at the same day supplied by a group of conceived female zebrafishes. The embryos for experiment were picked from the normally developed ones by microscope check, followed by subject to incubation experiments.

\section{Methods}

25 fertilized zebrafish eggs were set in each well with $5 \mathrm{~mL}$ of incubation solutions of $\mathrm{Ag}^{+}$, AgNPs@citrate, AgNPs@PVP and oxygenated water as normal incubation control at different concentrations $(0.1,0.2,0.5,0.8$, and $1 \mu \mathrm{g} / \mathrm{mL})$. The mortality was counted by microscopy observation at the time point of $24,48,72,96$ hours, respectively.

Table 1 . The experimental design

\begin{tabular}{|c|c|c|c|c|c|}
\hline Time point & Concentration & $\begin{array}{c}0.2 \\
{[\mu \mathrm{g} / \mathrm{mL}]}\end{array}$ & $\begin{array}{c}0.5 \\
{[\mu \mathrm{g} / \mathrm{mL}]}\end{array}$ & $\begin{array}{c}0.8 \\
{[\mu \mathrm{g} / \mathrm{mL}]}\end{array}$ & $\begin{array}{c}1 \\
{[\mu \mathrm{g} / \mathrm{mL}]}\end{array}$ \\
\hline $\begin{array}{c}\text { 24/48/72/96 } \\
{[\mathrm{h}]}\end{array}$ & $\begin{array}{l}\mathrm{Ag}^{+} \\
\text {AgNPs@citrate } \\
\text { AgNPs@PVP } \\
\text { Control }\end{array}$ & \multicolumn{4}{|c|}{ Mortality of embryos at respective time point } \\
\hline
\end{tabular}




\section{Results and discussion}

As shown in the TEM images of Fig.1 A and B, the majority of AgNPs prepared for this study were approximately spherical and basically uniform. The mean size of the particles is $\sim 15 \mathrm{~nm}$ by TEM image (Figure 1).

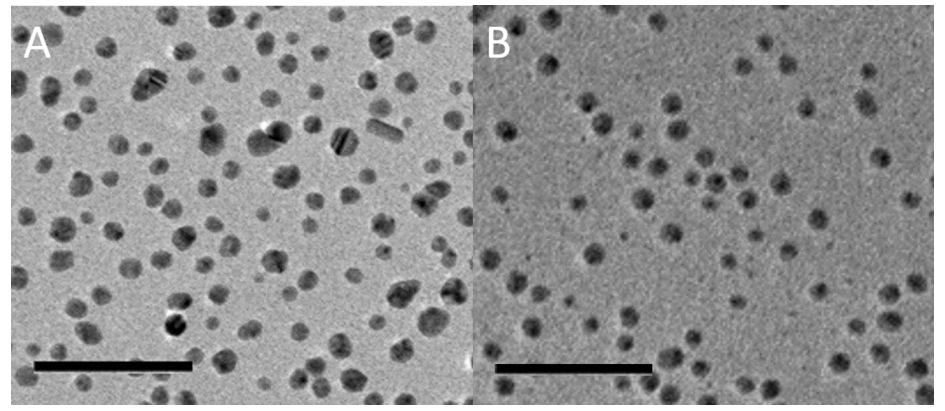

Figure 1. TEM images of AgNPs. (A) AgNPs@PVP; (B) AgNPs@ citrate. Scale bar: 100 nm.

After 96 hours observation, the numbers of death, hatching and malformation of zebrafish embryo in different groups were obtained. These data at different time points can be transferred to the following figures (from Figure 2 A to D).
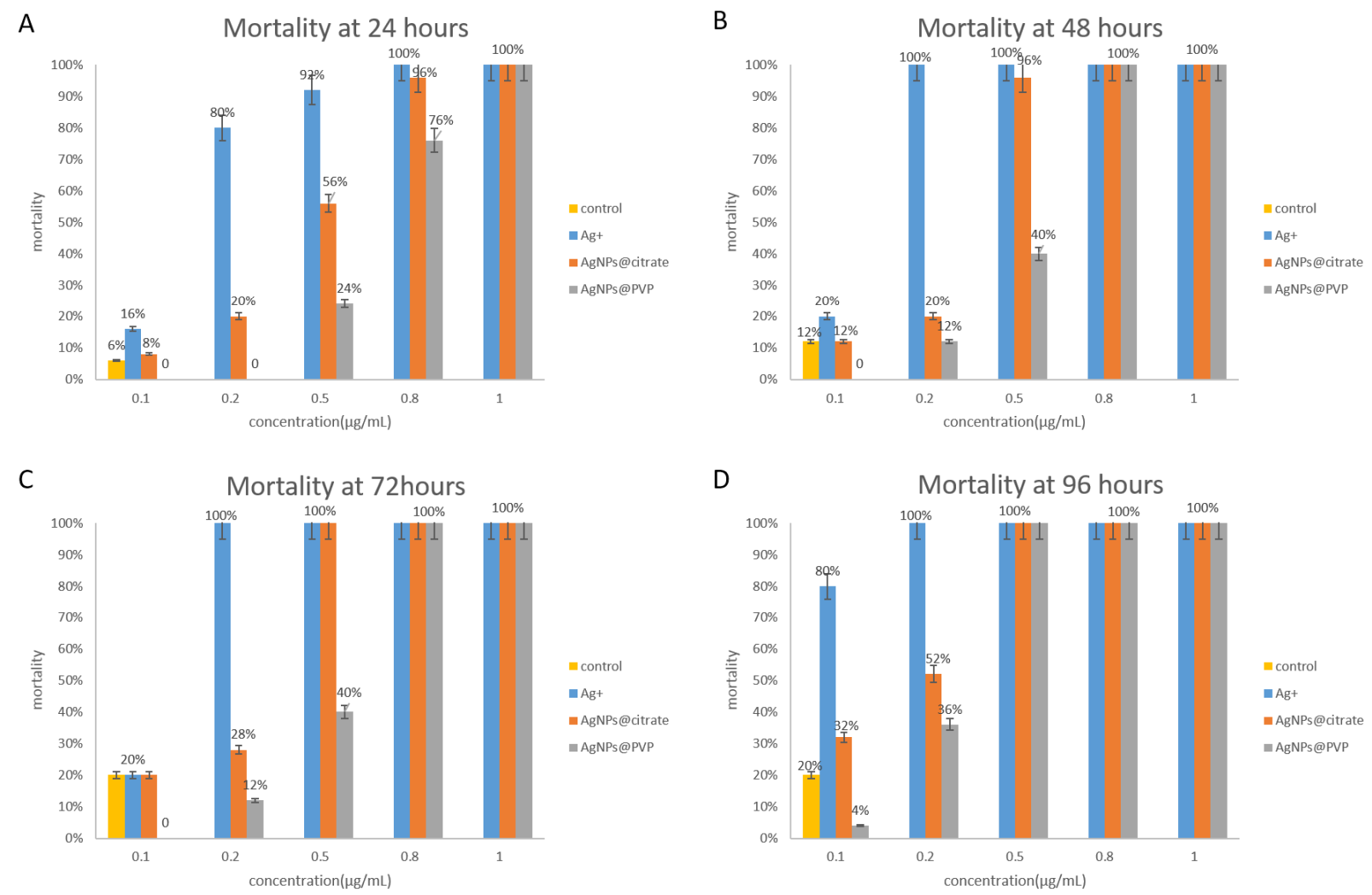

Figure 2. Mortality of embryos at different hours. (A) Mortality of embryos at 24 hours. (B) Mortality of embryos at 48 hours. (C) Mortality of embryos at 72 hours. (D) Mortality of embryos at 96 hours.

Based on the results shown above, it is obvious that Ag+ is most toxic. And AgNPs@PVP at 0.1 $\mu \mathrm{g} / \mathrm{mL}$ is least toxic to the embryonic development. At 48 hours, when the concentration of $\mathrm{Ag}^{+}$at $0.2 \mu \mathrm{g} / \mathrm{mL}$, the mortality of the zebrafish embryos was $100 \%$, and the death rate of the AgNPs@citrate was $20 \%$, which indicated that the toxicity of $\mathrm{Ag}^{+}$was significantly stronger than AgNPs@citrate. At 48 hours, the mortality rate of AgNPs@citrate was $96 \%$ at the concentration of 
$0.5 \mu \mathrm{g} / \mathrm{mL}$, while the mortality rates of AgNPs@PVP group was only $40 \%$. We speculate that the reason for such results may be the PVP prevents the release of the silver ions.

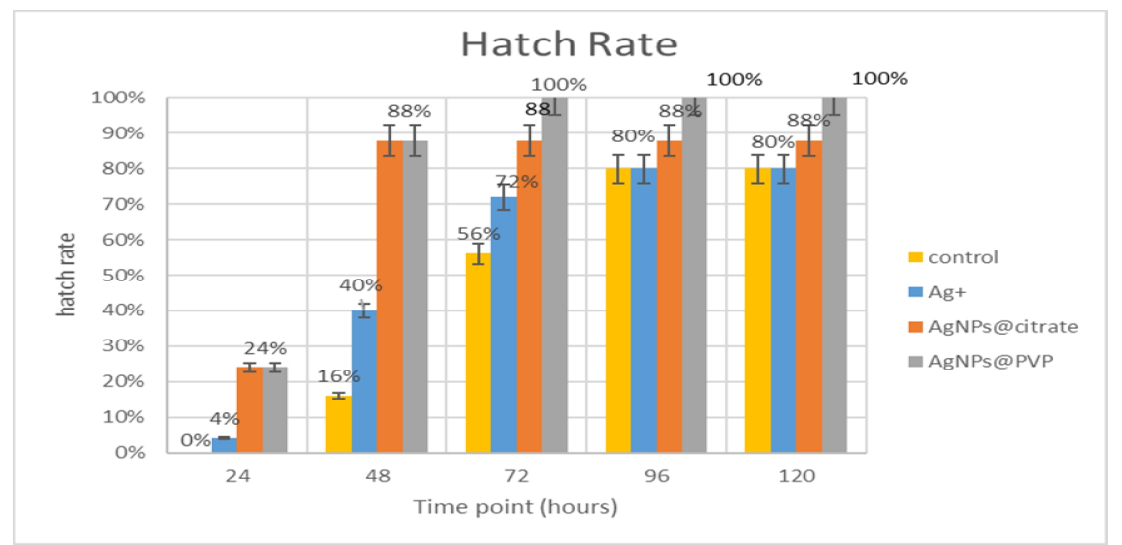

Figure 3. Hatching rate of embryos at the concentration of $0.1 \mu \mathrm{g} / \mathrm{mL}$

With regards to the hatching rate, Figure 3 shows that the hatching rate was $16 \%, 40 \%, 88 \%$ for control, $\mathrm{Ag}^{+}$, AgNPs@citrate and AgNPs@PVP group, respectively, at the concentration of 0.1 $\mu \mathrm{g} / \mathrm{mL}$ for 48 hours incubation. The result indicate that in the presence of $0.1 \mu \mathrm{g} / \mathrm{mL}$, AgNPs hardly cause any death of zebrafish embryos, AgNPs@citrate and AgNPs@PVP obviously accelerate the embryonic development as it takes five days to hatch in the control normal development. In addition, $\mathrm{Ag}^{+}$shows the higher toxicity when the $\mathrm{Ag}^{+}$and AgNPs@citrate at the same concentration. AgNPs@PVP is less toxic than AgNPs@ citrate at the same concentration.

Furthermore, the images of all kinds of the abnormal embryos through this study were recorded in the Figure 4 as a comparison.

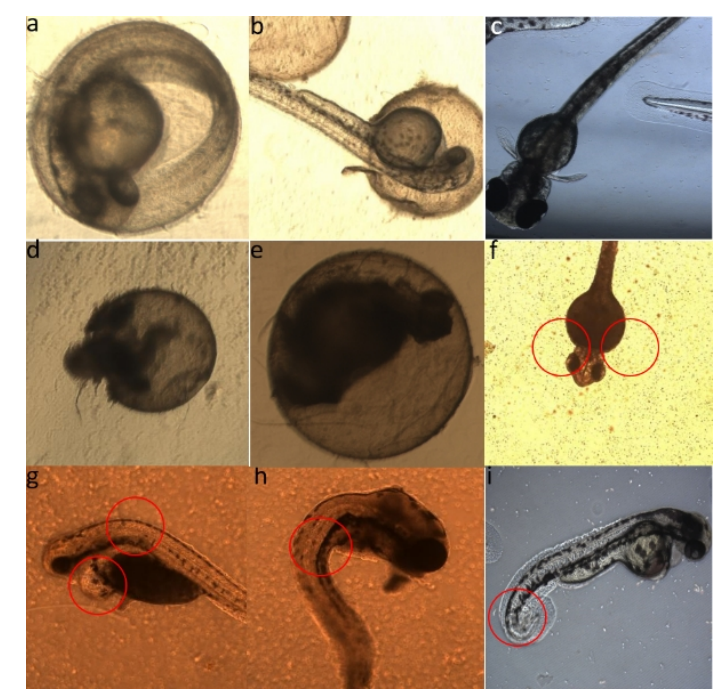

Figure 4. Microscopic images of all kinds of zebrafish embryos in experiments. (a) The normal unhatched embryo. (b) The normal hatching embryo. (c) The normal hatched embryo. (d) Dead Embryo due to embryonic fracture. (e) Dead embryo due to egg condensation. (f) The hatched juvenile fish without pectoral fins. (g) The juvenile fish with the edema of the pericardium. (h) The juvenile fish with the spine curvature. (i) The juvenile fish with tail bending. All the deformities were observed by incubation of specific $\mathrm{Ag}^{+}, \mathrm{AgNPs} @$ citrate or AgNPs@PVP.

\section{Conclusion and discussion}

In this study, the abnormalities induced by silver nanoparticles were investigated. When the embryo 
is exposed to silver nanoparticles, abnormal movement of the embryo can be observed. Previous research reported that nanoparticles have a direct effect on the central nervous system and the invasive nanoparticles can be distributed and remain in the brain during the early embryonic phase, interfering with central nervous system function and signal transduction [15]. When zebrafish embryos were treated with silver nanoparticles, the reduction of normal hatch is obvious. The direct effect of silver nanoparticles or silver ions also can lead to DNA damage and genetic abnormalities, whereas DNA damage and gene abnormalities can interfere with embryonic development, further leading to abnormal hatching rate [16].

In short, our experiments show that silver nanoparticles can lead to death and abnormality of zebrafish embryos although future efforts are still devoted to the understanding of the toxic mechanism by silver nanoparticle. Either silver ions or silver nanomaterials contribute the major toxicity to organisms, the main issue to be addressed here is that we need to control the release of untreated silver nanoparticles into the environment until we find an efficient way to tackle them.

\section{Acknowledgements}

This work was financially supported by the National Key Basic Research Program of China (2011CB933503) and high-level publication Foundation of Southeast of University (20161108)

\section{References}

[1] E. T. Hwang, J. H. Lee, Y. J. Chae, Y. S. Kim, B. C. Kim, B. I. Sang and M. B. Gu: Small Vol. 4 (2008), p. 746.

[2] W. K. Jung, H. C. Koo, K. W. Kim, S. Shin, S. H. Kim and Y. H. Park: Applied and Environmental Microbiology Vol. 74 (2008), p. 2171.

[3] S. Shrivastava, T. Bera, A. Roy, G. Singh, P. Ramachandrarao and D. Dash: Nanotechnology Vol. 18 (2007), p. 225103.

[4] M. Yamanaka, K. Hara and J. Kudo: Applied and Environmental Microbiology Vol. 71 (2005), p. 7589.

[5] S. Y. Liau, D. C. Read, W. J. Pugh, J. R. Furr and A. D. Russell: Letters in Applied Microbiology Vol. 25 (1997), p. 279.

[6] Z. Wang, S. Liu, J. Ma, G. Qu, X. Wang, S. Yu, J. He, J. Liu, T. Xia and G. B. Jiang: ACS Nano Vol. 7 (2013), p. 4171.

[7] Z. M. Xiu, Q. B. Zhang, H. L. Puppala, V. L. Colvin and P. J. Alvarez: Nano Letters Vol. 12 (2012), p. 4271.

[8] K. Bilberg, M. B. Hovgaard, F. Besenbacher and E. Baatrup: Journal of Toxicology Vol. 2012 (2011), p. 9.

[9] S. George, S. Lin, Z. Ji, C. R. Thomas, L. Li, M. Mecklenburg, H. Meng, X. Wang, H. Zhang, T. Xia and J. N. Hohman: ACS Nano Vol. 6 (2012), p. 3745.

[10]R. Nagel.: Altex Vol. 19 (2001), p. 38.

[11]J. Cheng, E. Flahaut and S. H. Cheng: Environmental Toxicology and Chemistry Vol. 26 (2007), p. 708.

[12]X. Zhu, L. Zhu, Z. Duan, R. Qi, Y. Li and Y. Lang: Journal of Environmental Science and Health Vol. 43 (2008), p. 278.

[13]H. Wu, J. Lin, P. Liu, Z. Huang, P. Zhao, H. Jin, J. Ma, L. Wen and N. Gu: Biomaterials Vol. 101 (2016), p. 9.

[14]H. Zhang and C. Zhang: Journal of Materials and Environmental Science Vol. 5 (2014), p. 231. 
[15]G. Oberdörster, Z. Sharp, V. Atudorei, A. Elder, R. Gelein, W. Kreyling and C. Cox: Inhalation Toxicology Vol. 16 (2004), p. 437.

[16]M. K. Yeo and M. S. Kang: Bulletin of the Korean Chemical Society Vol. 29 (2008), p. 1179. 